\title{
Photo-Induced Wettability Change in Films Containing Spiropyran Microcrystals
}

\author{
Shoichi HAYASHIDA, Hirotsugu SATO, ${ }^{*}$ and Shungo SugAwara \\ NTT Ibaraki Electrical Communication Laboratories, \\ Tokai, Ibaraki 319-11, Japan \\ * NTT Yokosuka Electrical Communication Laboratories, \\ Yokosuka, Kanagawa 238-03, Japan
}

(Received June 3, 1985)

\begin{abstract}
Photo-induced wettability changes in polymer films containing spiropyrans with long alkyl chains are investigated as a function of alkyl chain length and spiropyran content. It was found that spiropyrans with longer alkyl chains easily crystallize in polymer films, leading to a greater change in wettability. It was also found that the size of the formed microcrystals plays an important role in exhibiting photo-induced wettability changes. The wettability change disappeared after repeated photo-irradiation because stress caused by the change in molecular size pulverizes the microcrystals into smaller particles which have no influence on wettability change.

KEY WORDS Photo-Induced Wettability Change / Spiropyran Isomerization / Alkyl Chain / Polystyrene / Crystal / X-Ray Diffraction / Polarization Microscope /
\end{abstract}

Color changes in organic photochromic compounds are associated with reversible changes in the chemical structures of these compounds. Therefore, changes in physical properties other than the absorption spectra, for example, viscosity, solubility and wettability, are also simultaneously induced. ${ }^{1}$ Many studies on the active control of these properties by photo-irradiation have been carried out. ${ }^{2}$ Among them, photo-regulation of wettability is an attractive approach both from a practical point of view and scientific interest. This is because photo-regulated wettability makes it possible to obtain novel adsorbents for chromatography, ${ }^{3}$ printing media for repetitive use $\mathrm{e}^{4}$ and many other photo-regulable devices.

Typical polymers which show photo-induced wettability change include covalently bonded azobenzene, Malachite Green and the spiropyran group. ${ }^{3,5}$ Ishihara et al. studied the photo-induced change in wettability in copolymer films with various composition of $p$-phenylazoacrylanilide and hydroxyethyl methacrylate. Reversible change in dipole moment due to cis-trans isomerization of azobenzene moiety took place and change in wettability toward pure water corresponding to a change of 0.2 in $\cos \theta$ was obtained for poly( $p$-phenylazoacrylanilide). Negishi et al. ${ }^{5}$ studied the photoresponse of polystyrene with Malachite Green leucohydroxide side groups. In this film, a great change in wettability, from a level of hydrophobic poly(methyl methacrylate) to that of hydrophilic poly(vinyl alcohol), due to photo-dissociation was obtained. Change in wettability in the homopolymer film of spiropyran methacryl ester was studied also by Negishi et al. ${ }^{6}$ In this case, reversible change in wettability due to spiropyran-merocyanine conversion by photo-irradiation was observed.

In this work, long alkyl chains have been introduced into spiropyrans since the introduction of the long alkyl chains is useful for 
realizing novel properties in conventional material systems. The photo-induced changes in wettability of the polymer composites have been studied with respect to spiropyran content and alkyl chain length. This report also discusses the relationship between the obtained large wettability change and conditions on the polymer surface.

\section{EXPERIMENTAL}

Four spiropyrans (6-nitro-3', 3'-dimethylspiro-(2H-1-benzopyran-2,2'-indoline)) with different alkyl chain lengths (SP-1, -7, -12, -18 in Figure 1) as well as polystyrene (PSt), poly(methyl methacrylate) (PMMA) and poly(vinyl butyral) (PVBu) were used in this study. SP-1 was obtained from Tokyo Kasei Kogyo Co., Ltd, while SP-7 and SP-12 were purchased from Sogo Pharmaceutical Co., Ltd. SP-18 was prepared by a procedure similar to that reported by Gruda et al. ${ }^{7}$

PSt films, each containing a different spiropyran, were prepared by spin-coating a toluene solution containing the spiropyran and PSt on glass plates and then drying them at $40^{\circ} \mathrm{C}$ for one hour. Film thicknesses were $0.1-0.2 \mu \mathrm{m}$. The PMMA and PVBu films were prepared from a toluene solution and a toluene-ethanol (1:1) solution, respectively, by a similar procedure. The polymer concentration was $25 \mathrm{gl}^{-1}$ in each case.

The films were exposed to ultraviolet or visible light from a $500 \mathrm{~W}$ super high pressure mercury lamp (Ushio Denki) filtered through UV-D33S (ultraviolet light) and Y-50 (visible light) filters (Toshiba). The intensity of the ultraviolet light and the visible light were about $13 \mathrm{~mW} \mathrm{~cm}^{-2}$ and $24 \mathrm{~mW} \mathrm{~cm}^{-2}$, respectively. Ultraviolet irradiation time was $60 \mathrm{~s}$ and in this condition, coloration of the samples was almost saturated.

Contact angles for the films with distilled water were measured at room temperature using a contact angle goniometer (Erma). The contact angles were measured at four different positions on each film in order to obtain mean values. X-Ray diffraction measurements were carried out using an X-ray diffractometer (Geigerflex 2012, Rigaku Denki). The optical densities of the irradiated samples were measured using a spectrophotometer (Hitachi 303). Microscopic observation by polarized light was carried out with a VANOX Microscope (Olympus) and polarizer film.

\section{RESULTS AND DISCUSSION}

Spiropyrans have been considered not to isomerize in crystalline state so far. However, we found that spiropyrans with long alkyl chains shown in Figure 1 undergo photoisomerization. Figure 2 shows absorption spectra for an evaporated SP-18 film and for some polymer films containing SP-18. The Xray diffraction pattern for the evaporated SP18 film is shown in Figure 3, which indicates that the evaporated film was crystalline. The background is ascribed to the glass slide used as a substrate. Figure 2 clearly indicates that the evaporated film exhibited remarkable photochromism even in the crystalline state as well as the polymer films. Therefore, it should be noted that microcrystals of spiropyrons with long alkyl chains, which may be contained in the films as will be described later, undergo photo-isomerization.

Spectral reflectance curves for SP-7, -12, and -18 crystalline powders before and after irradiation are shown in Figure 4 (a). This figure indicates that SP-7 and -12 exhibit photochromism in the crystalline state as well as SP-18. In order to estimate the conversion ratio of spiropyran to merocyanine form, the reflectance curves were converted into absorption spectra. One for SP-18 is shown in Figure 4(b). Similar spectra were obtained for SP-7 and SP12. Krongauz et al. have reported that various spiropyran-merocyanine complexes as $\mathrm{AB}$ or $A_{n} B$ are formed besides merocyanine (B) when spiropyran (A) in polymer system is irradiated. ${ }^{8}$ The trapezoid characteristic of the peak in 


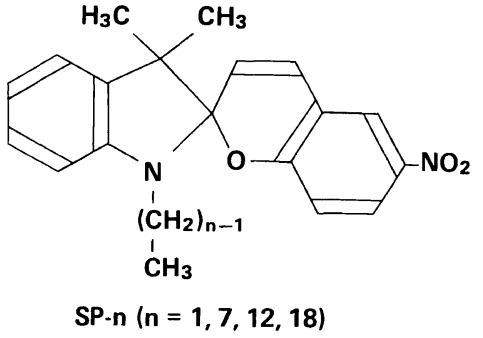

Figure 1. The structure of the spiropyrans with the long alkyl chain used.

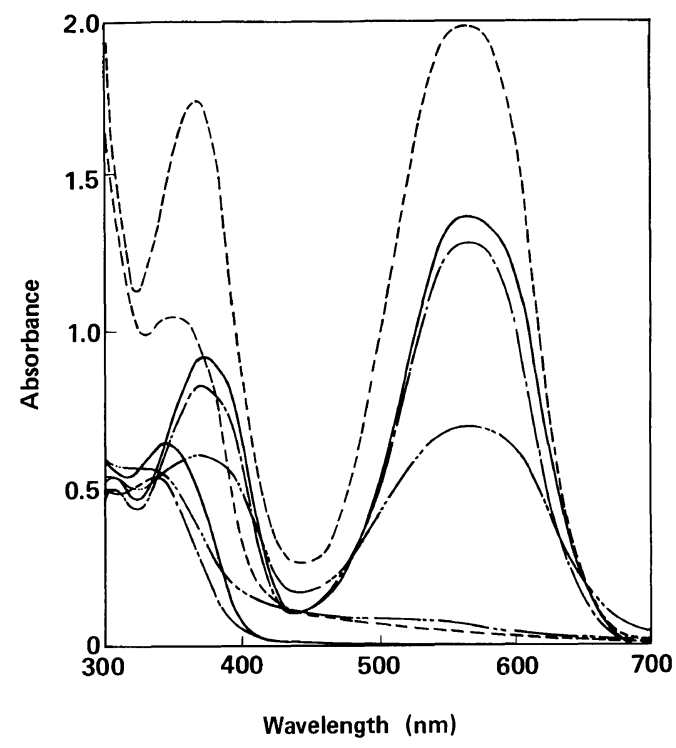

Figure 2. Absorption spectra for the films containing SP-18: —, $40 \mathrm{wt} \%$ in PSt; - - $40 \mathrm{wt} \%$ in PMMA; -- - $40 \mathrm{wt} \%$ in PVBu; ----, evaporated film.

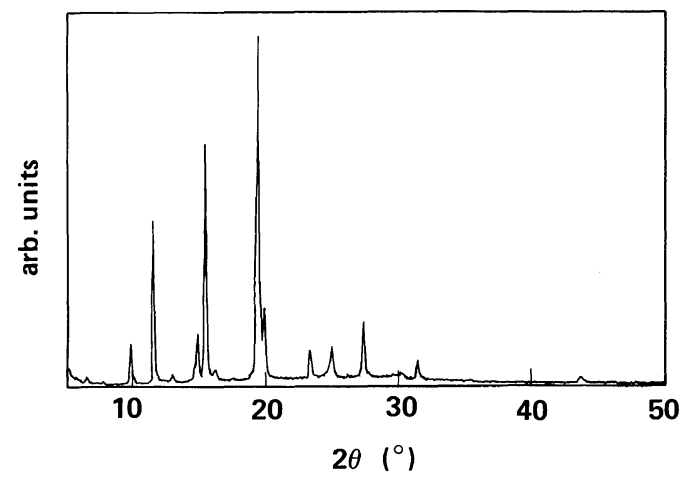

Figure 3. X-Ray diffraction pattern for the evaporated SP-18 film.

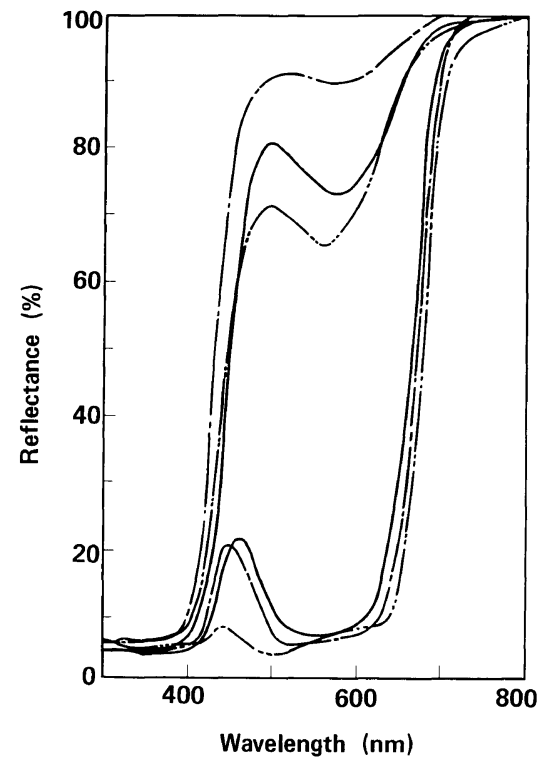

(a)

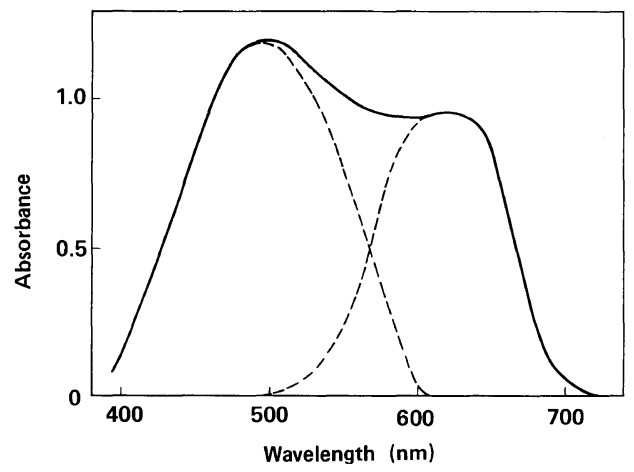

(b)

Figure 4. (a) Spectral reflectance curves for the microcrystalline SP- $n$ powders: ——, $n=7 ;-\ldots, n=12$; - - $n=18$. (b) Absorption spectrum converted from the spectral reflectance curve for SP-18 in (a).

Figure $4(\mathrm{~b})$ is ascribed to the plural components mentioned above. In comparison with the spectra reported by Krongauz et al., it is suggested that the peak in Figure 4(b) consists of that of $\mathrm{A}_{2} \mathrm{~B}$ and $\mathrm{AB}$. Molar extinction coefficients are about of the same order of $10^{4}-$ $10^{5}$ by Krongauz et al. ${ }^{8}$ There was almost no spiropyran molecules that did not take part in forming the spiropyran-merocyanine complex since the experimental condition such that 


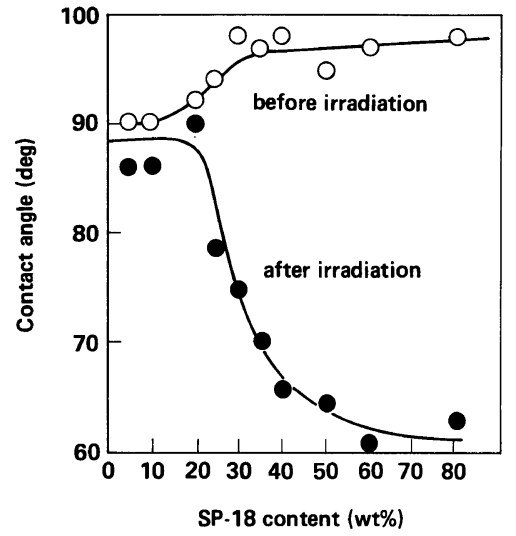

Figure 5. Effects of SP-18 content on contact angle. The binder was polystyrene. The net irradiation energy was $780 \mathrm{~mJ} \mathrm{~cm}^{-2}$.

absorbance was saturated was used in the present study. Therefore, it is estimated that nearly $40 \%$ of the spiropyran molecules were converted to merocyanine form on the surface of the microcrystals formed in the films when taking into account the absorption intensity and molar extinction coefficients of the two components.

Figure 5 shows changes in photo-induced wettability on the PSt films containing SP-18. When the SP-18 content was below $20 \mathrm{wt} \%$, there was almost no change in the contact angle. Above that content, however, a change in the contact angle appeared both before and after ultraviolet irradiation. For the samples prior to ultraviolet irradiation, the contact angle increased to $98^{\circ}$ with content increase. For the samples after irradiation, the contact angle decreased steeply at a content above $20 \mathrm{wt} \%$ and reached $61^{\circ}$ at $60 \mathrm{wt} \%$. The net contact angle change at $60 \mathrm{wt} \%$ was $37^{\circ}$, corresponding to a change of 0.62 in $\cos \theta$. The results for the SP-1, -7 , and -12 in the PSt films are shown in Figure 6. In the case of SP-1, there was no change below $50 \mathrm{wt} \%$ while slight change was observed above $50 \mathrm{wt} \%$. In the case of SP-7 and SP-12, changes were observed above about $40 \mathrm{wt} \%$. However, the magnitude was less than that for SP-18.

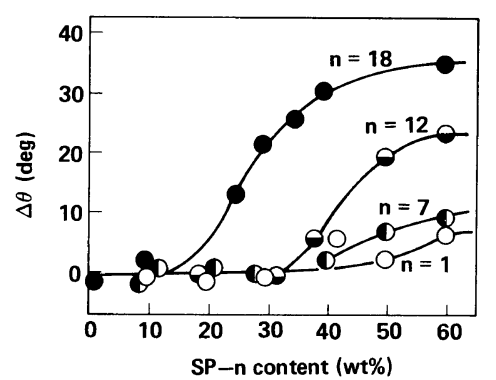

Figure 6. Effect of SP- $n$ content on contact angle change. Polymer: PSt.

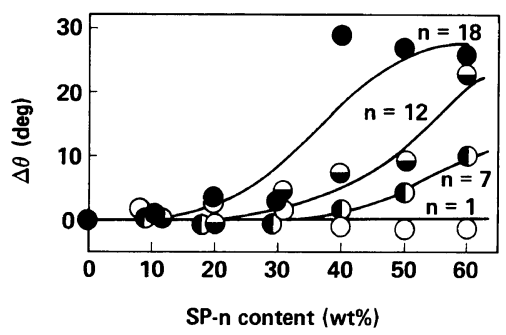

Figure 7. Effect of SP- $n$ content on contact angle change. Polymer: PMMA.

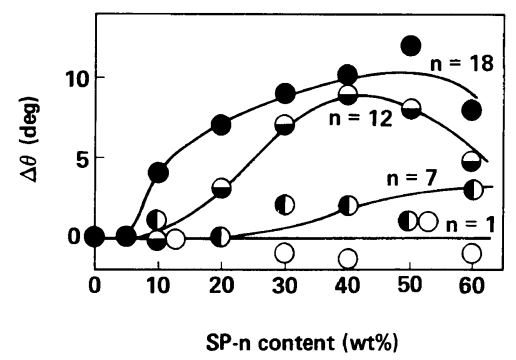

Figure 8. Effect of SP- $n$ content on contact angle change. Polymer: PVBu.

Results for PMMA and PVBu films are shown in Figures 7 and 8. In these films, the change in the contact angle was smaller than in the PSt films as a whole. This is because PMMA and PVBu are relatively hydrophilic and their contact angles with water are originally smaller than that of PSt; the contact angles for PMMA and PVBu were $65^{\circ}$ and $72^{\circ}$, respectively. However, the tendency that a larger change in wettability is obtained for a longer $N$-alkyl chain is in common with the case for PSt films. The actual number of 

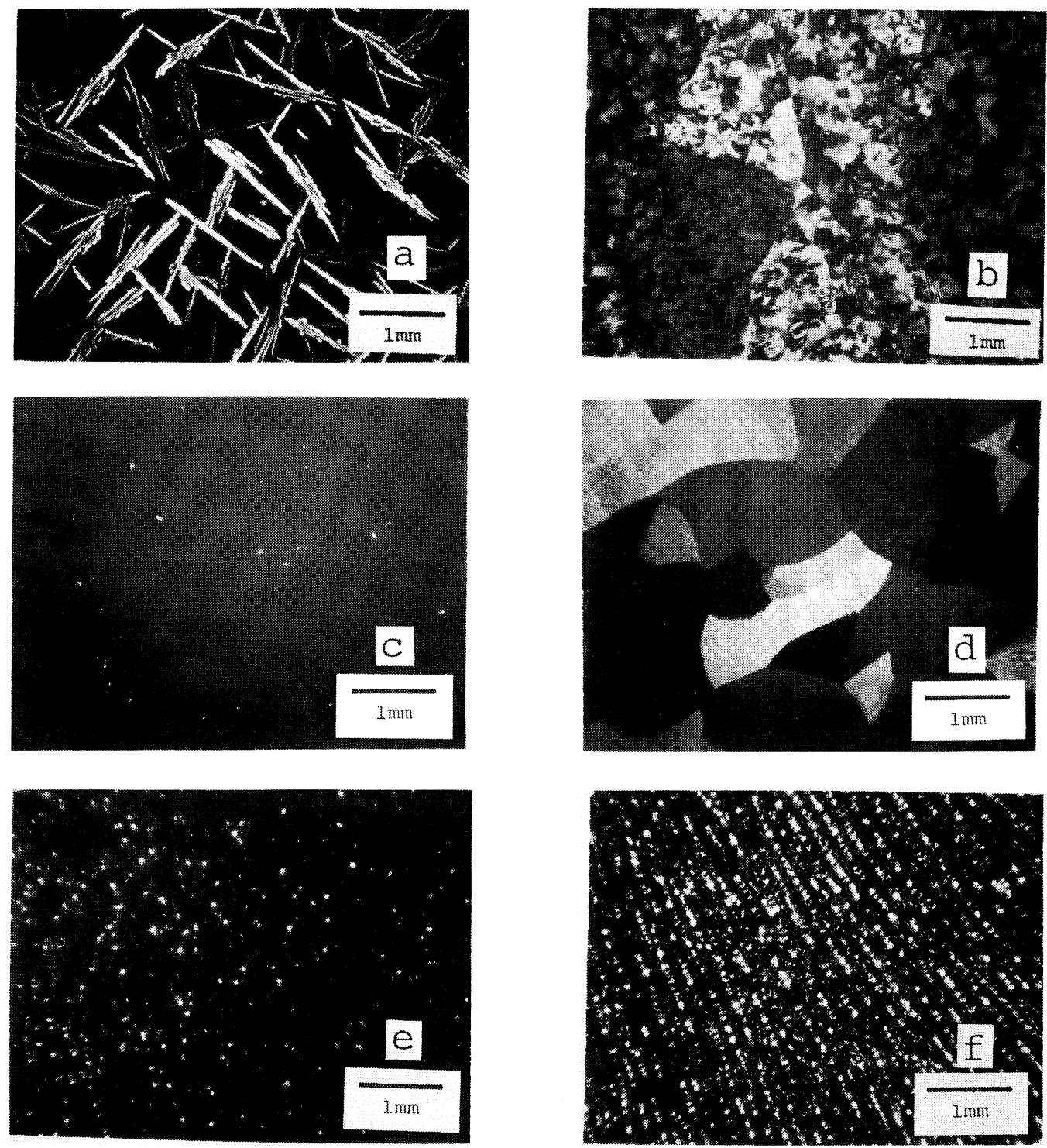

Figure 9. Polarization microphotographs for the films containing SP-18. (a) $30 \mathrm{wt} \%$ in PSt, (b) $40 \mathrm{wt} \%$ in PSt, (c) $30 \mathrm{wt} \%$ in PMMA, (d) $40 \mathrm{wt} \%$ in PMMA, (e) $30 \mathrm{wt} \%$ in PVBu, (f) $40 \mathrm{wt} \%$ in PVBu.

spiropyran molecules per unit weight is smaller when the alkyl chain becomes longer. Taking this into consideration, it is noteworthy that the magnitude of the change in wettability is not exclusively dependent upon the number of dissociative spiropyran skeletons.

Polarization microphotographs for some of the films containing SP-18 are shown in Figure
9. A number of needles about $1 \mathrm{~mm}$ in length formed in the PSt film with $30 \mathrm{wt} \%$ of SP-18 and plates of $0.2-0.3 \mathrm{~mm}$ in width formed in the case of $40 \mathrm{wt} \%$ film. In the change in wettability shown in Figure 6, the change for the $30 \mathrm{wt} \%$ film was in a transient range and that for $40 \mathrm{wt} \%$ film was in a plateau range, as is consistent with the amount of microcrystals 
shown in Figures 9 (a) and 9(b).

The relationship between microcrystal amount and change in wettability is similar in the case of PMMA films. A trace amount of microcrystals for $30 \mathrm{wt} \%$ film and a number of plates in whole sample for $40 \mathrm{wt} \%$ film were observed in Figures 9 (c) and 9 (d). This is in close agreement with the result that little change in wettability was observed in the $30 \mathrm{wt} \%$ film and that a great change was obtained for the $40 \mathrm{wt} \%$ film.

The PVBu films were somewhat different. In Figure 9 (f), microcrystals are observed along the direction in which the sample solution runs in the spin-coating procedure. This is presumably a result of low miscibility of PVBu with SP-18. However, this phenomenon was not remarkable for the $30 \mathrm{wt} \%$ film. The PVBu films of long alkyl chain spiropyran content above $40 \mathrm{wt} \%$ have little smoothness probably because of the low miscibility. The roughness of the film surface may lead to capillary action. Therefore, it is difficult to interpret the change in wettability only by the existence of the microcrystals in this instance.

In contrast to the films mentioned above, no crystals formed in films which exhibited no change in wettability, for example, in the PSt films containing SP-18 below $20 \mathrm{wt} \%$, SP-12 below $30 \mathrm{wt} \%$ and SP-1 below $40 \mathrm{wt} \%$. Properties of the film surface are accordingly influenced by some of the microcrystals beneath it. These results suggest that microcrystals on the polymer surface play an important role for these films to indicate changes in wettability. In other words, a spiropyran domain as large as that of a microcrystal is necessary for change in wettability. The difference in the morphology of microcrystals, such as needles and plates shown in Figure 9, may also affect wettability. However, it cannot be considered separately from the size of microcrystals. The significance of the role of the microcrystals is supported by the fact that hydrophilic and hydrophobic functions of amphiphilic polymers are largely influenced by the size and geometry of hydrophilic and hydrophobic domains, as reported by Okano et al. ${ }^{9}$

In order to more thoroughly investigate the relationship between these microcrystals and the change in wettability, X-ray diffraction measurements were performed. X-Ray diffraction patterns for PSt films containing various amounts of SP-18 are shown in Figure 10 (a). There was no diffraction from the film when the SP -18 content was at a $10 \mathrm{wt} \%$ level. This suggests that SP-18 is molecularly dispersed in the film. Diffraction appeared, however, when the SP-18 content increased to $20 \mathrm{wt} \%$. Moreover, the diffraction intensity increased as the SP- 18 content increased. This suggests that SP18 molecules cannot separately exist above this content and that microcrystals begin to form. Figure 10 (b) shows X-ray diffraction patterns for the PMMA and the PVBu films each containing $40 \mathrm{wt} \%$ of SP-18. It is obvious from this figure that microcrystals formed in both of the films. In addition, the diffraction angles observed for the PMMA and the PVBu films coincide with those of the PSt film, indicating that the characteristics of the microcrystals in the PMMA and the PVBu films are identical to those in the PSt film. This is supported also by the above results that the smaller change in wettability in the PMMA and the PVBu films is ascribed to the higher hydrophilicity of the matrix polymers themselves rather than the difference in the isomerization conversion or the arrangement of the spiropyran.

Figure 11 shows a relationship between SP18 content in PSt film and the intensity of the net diffraction. It is clear from this figure that the diffraction intensity shows a sharp increase beginning at about $20 \mathrm{wt} \%$. The point of onset coincides exactly with the point at which the wettability change begins as shown in Figure 5. In addition, the two curves, diffraction intensity and contact angle change vs. SP-18 content, also correspond above $20 \mathrm{wt} \%$. This clearly indicates that the microcrystals formed on the polymer surface are largely responsible 


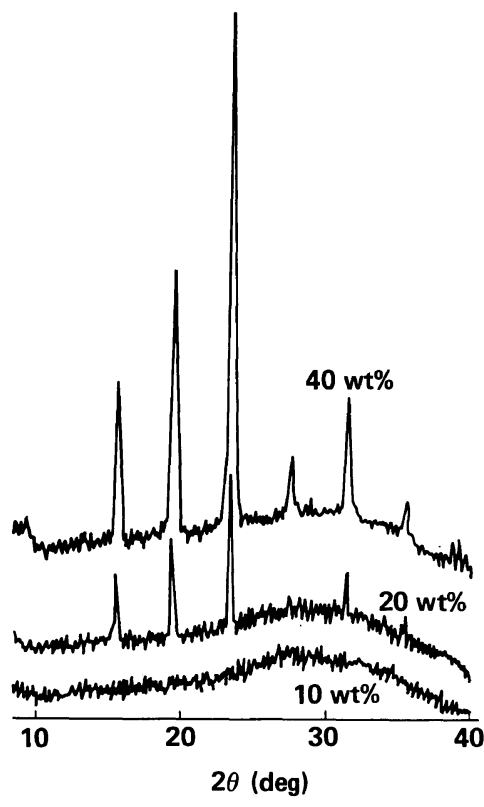

(a)

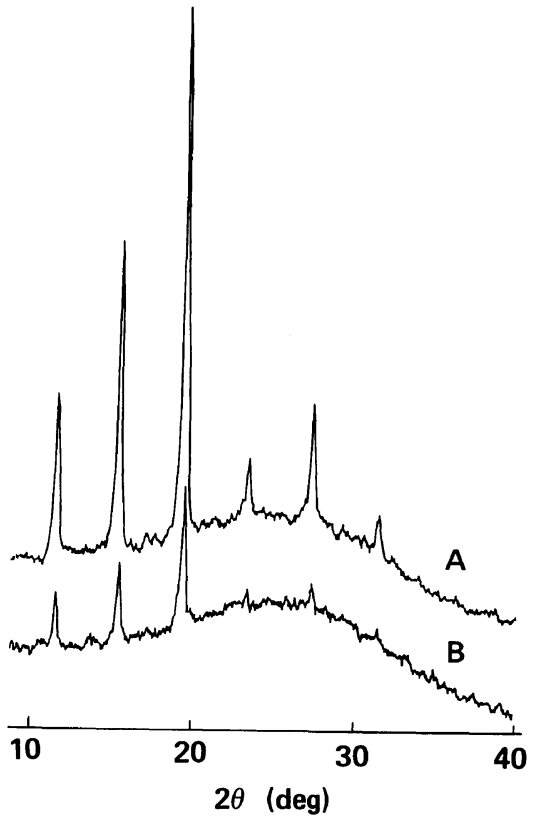

(b)

Figure 10. X-Ray diffraction from the films containing SP-18. (a): polymer, PSt: film thicknesses, $0.1-0.2 \mu \mathrm{m}$. (b): A, $40 \mathrm{wt} \%$ in PMMA; B, $40 \mathrm{wt} \%$ in PVBu.

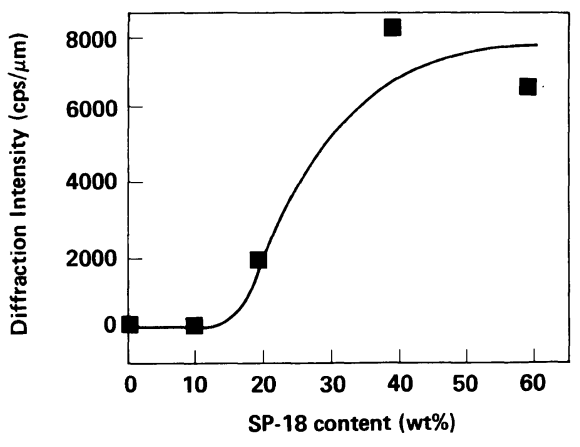

Figure 11. Effect of SP-18 content on the X-ray diffraction intensity: polymer, PSt.

for the change in wettability.

Microcrystals of spiropyrans with longer alkyl chains are more easily formed, leading to greater change in wettability. The solubility of spiropyrans in the polymer films is considered the reason why longer alkyl chains lead to crystallization in spite of the smaller number of spiropyran molecules. Spiropyrans them- selves have high solubility in polymers. In fact, when much SP-1 is dissolved in a polymer such as PSt or PMMA, a homogeneous, transparent film is obtained. However, a long alkyl chain gives polyethylene-like characteristics to a spiropyran molecule because polyethylene can be considered to be a very long alkyl chain. Since polyethylene can scarcely be dissolved in any solvent, the solubility of spiropyran with long alkyl chains in a polymer such as PSt or PMMA is low. This suggests that phase separation of spiropyrans with polyethylene-like characteristics is easily induced.

Figure 12 shows changes in wettability as well as the optical densities of PSt film with a $49 \mathrm{wt} \%$ SP-18 content when ultraviolet and visible light were alternately and repeatedly irradiated. The change in the contact angle was about $40^{\circ}$ for the first ultraviolet irradiation. However, the initial contact angle could not be recovered by visible light irradiation. The change in contact angle decreased gradually in 


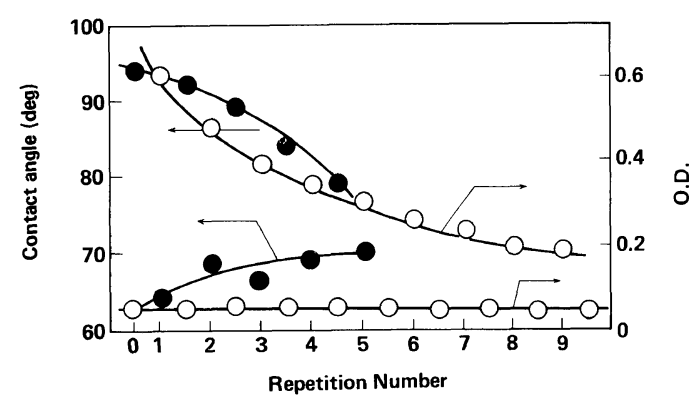

Figure 12. Effect of repeated ultraviolet and visible light irradiation on contact angle (O) and optical density (O) at $580 \mathrm{~nm}$ where the merocyanine form is absorptive. The upper $\bigcirc$ and the lower $\bigcirc$ are for the samples exposed to the ultraviolet light. The lower $\bigcirc$ and the upper are for the samples exposed to the visible light.

the course of repetitive photo-irradiation, and became negligible after five repetitions. On the other hand, coloration fatigue proceeded more slowly. Coloration fatigue in a spiropyran system is attributed to changes in spiropyran at a molecular level such as side reactions or conversion to non-photoresponsive isomers. In the present study, however, the change in the contact angle was not in agreement with the coloration fatigue profile. This suggests that the decrease in the wettability change is not attributable to the fatigue at the molecular level but rather to some other factor mentioned below.

A polarization microphotograph of a PSt film with a $40 \mathrm{wt} \%$ SP-18 content is shown in Figure 13. Since this sample was exposed to ultraviolet and then visible light irradiation, it exhibited a smaller change in wettability than that for a virgin sample, as shown in Figure 9. Microphotograph of a film before irradiation is shown in Figure $9(\mathrm{~b})$. The size of the microcrystals after photo-irradiation is obviously smaller than before photo-irradiation. This is due to the stress which is brought about by changes in molecular size caused by photoisomerization. An increase in the area occupied by molecules has been confirmed by surface pressure changes in the spiropyran monolayer at the air-water interface. ${ }^{10}$ Stress thus induced

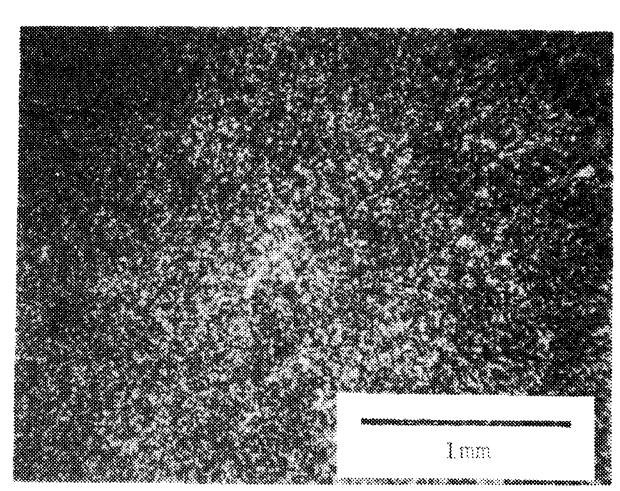

Figure 13. Polarization microphotograph for SP-18 $(40 \mathrm{wt} \%) /$ PSt film after ultraviolet and then visible light irradiation.

distorts and pulverizes the microcrystals into further smaller particles. As mentioned before, microcrystal size is important for the films to exhibit a change in wettability. Therefore, it is suggested that the microcrystal size decreases by the stress so that the spiropyran domain cannot contribute to the change in wettability any longer. Presumably resulting spiropyran microcrystals scattered in the films are incapable of forming a successive water layer on them.

In conclusion, changes in the photo-induced wettability of polymer films containing long chain spiropyrans were investigated and the following results obtained: 1) A $37^{\circ}$ change in the contact angle (from $98^{\circ}$ to $61^{\circ}$ ) can be obtained by an ultraviolet irradiation of about $800 \mathrm{~mJ} \mathrm{~cm}^{-2}$; 2) the formation of microcrystals is found to be essential for changing wettability from polarization microscopic observations and X-ray diffraction measurements; 3) the disappearance of the change in wettability occurs because internal stress involved in repetitive photo-isomerization results in pulverization of the microcrystals.

\section{REFERENCES}

1. M. Irie, "Molecular Models of Photoresponsiveness," G. Montagnoli and B. F. Erlanger, 
Ed., Plenum Press, New York, N. Y., 1983, p 291.

2. For a review see the book containing ref 1 and those cited therein.

3. K. Ishihara, A. Okazaki, N. Negishi, I. Shinohara, T. Okano, K. Kataoka, and Y. Sakurai, J. Appl. Polym. Sci., 27, 239 (1982).

4. M. Irie, Kogyo Zairyo, 31, 65 (1983).

5. N. Negishi, K. Tsunemitsu, and I. Shinohara, Polym. J., 13, 411 (1981).

6. N. Negishi, K. Tsunemitsu, K. Ishihara, I.
Shinohara, T. Okano, K. Kataoka, T. Akaike, and Y. Sakurai, Kobunshi Ronbunshu, 37, 287 (1980).

7. I. Gruda and R. M. Leblanc, Can. J. Chem., 54, 576 (1976).

8. V. A. Krongauz and E. S. Goldburt, Macromolecules, 14, 1382 (1981).

9. T. Okano, M. Katayama, and I. Shinohara, J. Appl. Polym. Sci., 22, 369 (1978).

10. E. E. Polymeropoulos and D. Möbius, Ber. Bunsenges. Phys. Chem., 83, 1215 (1979). 